\title{
Stellar Observations Network Group: The prototype is nearly ready
}

\author{
Frank Grundahl ${ }^{1}$, Jørgen Christensen-Dalsgaard ${ }^{1}$, Pere L. Pallé P, $^{2,3}$, \\ Mads F. Andersen ${ }^{1}$, Søren Frandsen ${ }^{1}$, Kennet Harpsøe $\mathbf{e}^{4,5}$, \\ Uffe Gråe Jørgensen ${ }^{4,5}$, Hans Kjeldsen ${ }^{1}$, Per K. Rasmussen ${ }^{3}$, \\ Jesper Skottfelt ${ }^{4,5}$, Anton N. Sørensen ${ }^{4}$, and Andrea Triviño Hage ${ }^{2,3}$ \\ ${ }^{1}$ Stellar Astrophysics Centre, Aarhus University, Ny Munkegade 120, \\ 8000 Aarhus C, Denmark, email: fgj@phys.au.dk \\ ${ }^{2}$ Instituto de Astrofísica de Canarias (IAC), E-38200 La Laguna, Tenerife, Spain \\ ${ }^{3}$ Dept. Astrofísica, Universidad de La Laguna (ULL), E-38206 Tenerife, Spain \\ ${ }^{4}$ Niels Bohr Institute, University of Copenhagen, Juliane Mariesvej 30, \\ 2100 København $\varnothing$, Denmark \\ ${ }^{5}$ Centre for Star and Planet Formation, Natural History Museum, \\ University of Copenhagen, Østervoldgade 5-7, 1350 København K, Denmark
}

\begin{abstract}
The prototype telescope and instruments for the Stellar Observations Network Group (SONG) are nearing completion at the Observatorio del Teide on Tenerife. In this contribution we describe the current status (autumn 2013) of the telescope and its instrumentation. Preliminary performance characteristics are presented for the high-resolution spectrograph based on daytime observations of the Sun and a 4 hour test series obtained for the sub-giant $\beta$ Aquilae.
\end{abstract}

Keywords. instrumentation: spectrographs, techniques: radial velocities, Sun: oscillations, stars: oscillations

\section{Introduction}

During the past 10 years the field of asteroseismology has undergone a virtual revolution in the amount and quality of the data which have become available. This is primarily due to space-based missions such as WIRE (Buzasi 2004), MOST (Matthews et al. 2004) CoRoT (Baglin et al. 2012) and Kepler (Chaplin \& Miglio 2013). In particular CoRoT and Kepler have provided exciting new results for a wide range of stars and stellar types. In common for all these missions is that the stellar oscillations are observed as intensity variations (photometry). The first ground-based detections of solar-like oscillations used spectroscopy to determine variations in radial velocity or equivalent width of Balmer lines (Kjeldsen et al. 2003). The ground-based efforts to detect the oscillations via photometry were largely unsuccessful due to the effects of the Earth's atmosphere. It is well known that for solar-like oscillators radial-velocity measurements have lower background levels (Harvey 1988) and that low-frequency and $l=3$ modes can be detected more easily than for intensity measurements (Dziembowski 1977, Harvey 1988).

The Stellar Observations Network Group (SONG) is an initiative to develop a groundbased network of $1 \mathrm{~m}$-class telescopes which makes it possible to obtain high-quality oscillation measurements for the brightest solar-like oscillators in the sky. The second driver for SONG is to carry out studies of extra-solar planets using the microlensing method by following up on real-time triggers from large surveys such as OGLE $\dagger$ and MOA $\ddagger$ In

$\dagger$ http://ogle.astrouw.edu.pl/ogle4/ews/ews.html

$\ddagger$ http://www.phys. canterbury.ac.nz/moa/index.html 
this contribution we describe the component of SONG related to asteroseismology - its design, instruments and status of the network efforts.

\section{The SONG node at Tenerife}

For the development of the network to proceed as smoothly as possible it was decided to complete the construction of a full prototype network node before requesting funding for more nodes such that the feasibility could be determined.

\subsection{Telescope}

Based on the results by Butler et al. (2004) from UVES we found by scaling of their results that a telescope of $1 \mathrm{~m}$ diameter would be sufficient for the detection of oscillations in bright stars. The telescope is carried on an alt-az mount and driven by magnetic-torque motors which allow fast re-pointing (speeds up to $20^{\circ}$ per second). It is a very compact design ensuring a very stiff structure which gives a measured pointing model performance of $3^{\prime \prime}$ with a pointing model based on only 30 stars. The telescope is housed in a $5 \mathrm{~m}$ diameter dome which has been insulated to limit thermal buildup during daytime - at present the insulation does not provide the desired reduction in dome temperature but a cooling unit will be installed to remedy it.

At the time of writing (September 2013) the control of the active primary mirror, which is only $5 \mathrm{~cm}$ thick, is undergoing final tests. The short-term wavefront reconstruction and control is working very well and delivers a nearly diffraction limited performance of the telescope optics based on Shack-Hartmann measurements of bright stars. The Coudé path will undergo final alignment during the autumn once the work on the active optics is completed.

While not fully commissioned yet, our tests with remote observations show that we can acquire and observe objects over the whole sky with fully automatic scripts and operating remotely.

The two instruments for SONG consist of a high-resolution optical spectrograph mounted at the telescope Coudé focus to obtain high precision radial velocities and two EMCCD cameras mounted at one of the two Nasmyth foci. The EMCCD cameras are used for high spatial-resolution photometry and lucky imaging (see for example Harpsøe et al. 2012a).

\subsection{Spectrograph}

In order to facilitate precision radial-velocity measurements, a high-resolution spectrograph is needed. The SONG spectrograph is a "classical" cross-dispersed, white pupil, echelle spectrograph with a design inspired by UVES and HARPS. The basic parameters for the spectrograph are provided in Table 1. The spectrograph is located in a standard, insulated shipping container next to the telescope pier. The legs for the spectrograph optical table are secured to a concrete foundation which is not in mechanical contact with the container or telescope foundations. Inside the container are the control computers, power supplies, and calibration lamps for the spectrograph which itself is housed in an insulated, temperature regulated box.

Inspired by the excellent results by Butler et al. (2004) we decided to used the iodine method for the determination of the precise radial velocities required for asteroseismic studies. We employ an iodine cell of $10 \mathrm{~cm}$ length and $5 \mathrm{~cm}$ diameter, which is operated by a ThorLabs TC200 controller and kept at a temperature of $65^{\circ} \mathrm{C}$ to ensure full evaporation of the iodine. For the calculation of radial velocities a high-resolution template of the 
Table 1. Characteristics of the SONG spectrograph

\begin{tabular}{|c|c|}
\hline Resolution $^{1}$ & $35000-112000$ \\
\hline Pixel scale (average) $)^{1}$ & $0.02 \AA$ \\
\hline Wavelength range & $4400-6900 \AA$ \\
\hline Number of spectral orders ${ }^{2}$ & 51 \\
\hline Collimated beam diameter & $75 \mathrm{~mm}$ \\
\hline Collimator focal ratio & $\mathrm{F} / 6$ off-axis parabola \\
\hline Echelle grating & Newport, R4, $31.6 \mathrm{l} / \mathrm{mm}, 9 \times 33 \mathrm{~cm}^{2}$ ruled area \\
\hline Detector & Andor, IKon-L, $2 \mathrm{~K} \times 2 \mathrm{~K}$ \\
\hline Readout speed/time & $3 \mathrm{MHz}, 2.3 \mathrm{~s}$ full-frame \\
\hline Number of slits & 6 \\
\hline Slit length & $10^{\prime \prime}$ \\
\hline Order separation & 24 pixels (bluest) and 7 pixels (reddest) \\
\hline
\end{tabular}

Notes:

${ }^{1}$ Average value over all orders. The resolution and pixel scale varies slightly across the detector area. The reddest orders have resolutions up to 125000 .

${ }^{2}$ Only orders with wavelengths shorter than $5250 \AA$ are fully covered by the detector. The camera has been designed to cover the orders fully with a larger detector.

iodine absorption spectrum is needed. This was obtained using the Fourier Transform Spectrometer at Lund Observatory.

In addition to light from the telescope it is possible to feed the spectrograph with light from a ThAr lamp for wavelength calibration and a halogen lamp for flat fielding. Furthermore it was decided at an early stage to provide also the possibility to observe the Sun by pointing the telescope to the blue daytime sky (looking through a dome window). It was, however, found during laboratory tests that an optical fibre pointing directly to the Sun provided a very high flux - allowing exposure times up to $0.5 \mathrm{~s}$ through the iodine cell without saturating the detector. Our current setup comprises a standard, circular, optical fibre from ThorLabs with a core diameter of $400 \mu \mathrm{m}$ giving a fully illuminated slit. Outside the container the entrance of the fibre is mounted on a solar tracker ensuring pointing to the Sun. In order to provide scrambling of the light before it enters the fibre we have mounted a piece of grey masking tape $1 \mathrm{~cm}$ in front of the fibre. The results from daytime solar observations are described below.

\subsection{Lucky-imaging camera}

The second main science goal for SONG is to study exoplanets via the microlensing method, requiring that precise photometry can be obtained in the crowded fields towards the Galactic bulge where most such events occur. Photometry in crowded fields benefits greatly from high spatial resolution and to allow for this a two-colour lucky imaging instrument is placed at the Nasmyth focus of the telescope. Via a dichroic beamsplitter (split at $6500 \AA$ ) light is directed towards two identical science cameras allowing simultaneous imaging in two bands. In addition, each channel has a filter wheel. The cameras are Andor iXon model 897, which can run at full frame speeds of up to $33 \mathrm{~Hz}$ - in this way we can carry out lucky imaging. 
The field of view for the cameras is $46^{\prime \prime}$ on a side, providing a 0.09 pixel scale in order to provide $\sim 2$ pixel sampling of the telescope point-spread function at a wavelength of $800 \mathrm{~nm}$. The on-sky performance of the cameras has not yet been tested. However, a nearly identical camera setup (same camera and pixel scale) has been established at the Danish $1.54 \mathrm{~m}$ telescope in La Silla and results of test observations can be found in Harpsøe et al. (2012b).

\section{Spectroscopic measurements}

With the prototype telescope nearly operational and a dedicated fibre-feed for solar observations it has been possible to carry out limited tests of the velocity precision that can be obtained with the spectrograph.

\subsection{Solar observations}

During June 11-16, 2012, we carried out a solar-observing experiment. Each morning of the 6 days the input end of the fibre was pointing towards the Sun and the tracker started. We collected approximately 12500 spectra each day, totalling nearly 70000 spectra. The data were reduced using a dedicated pipeline written in IDL (see Triviño Hage et al. 2012). As mentioned previously, the exposure time was set at $0.5 \mathrm{~s}$ to avoid saturation in the wavelength region covered by the iodine absorption lines $(5000-6200 \AA)$. In conjunction with a $2.3 \mathrm{~s}$ readout time this led to a sampling rate of $2.81 \mathrm{~s}$ between each successive spectrum. Each day, before starting the solar observations, calibration frames were acquired, consisting of 120 flat fields, 120 bias images and 3 ThAr spectra. In addition, in order to construct a solar template spectrum needed for the velocity extraction, 50 solar spectra without the iodine cell were acquired followed immediately by 50 flat fields through the iodine cell - these were used to determine the spectrograph pointspread function. A detailed account of the observations required for the iodine method was provided by Butler et al. (1996). All the spectra were obtained with a resolution of 120000 .

A short segment of the time-series is shown in Fig. 1. The well known 5-minute solar oscillation is easily seen in the data - a clear indication that the measurement precision is very high. We have computed the power spectrum for the entire series - see Fig. 2 . An analysis of the high-frequency noise indicated an average measurement precision of $0.8 \mathrm{~m} \mathrm{~s}^{-1}$ per point, with some degradation when the Sun is low in the sky, in late afternoon.

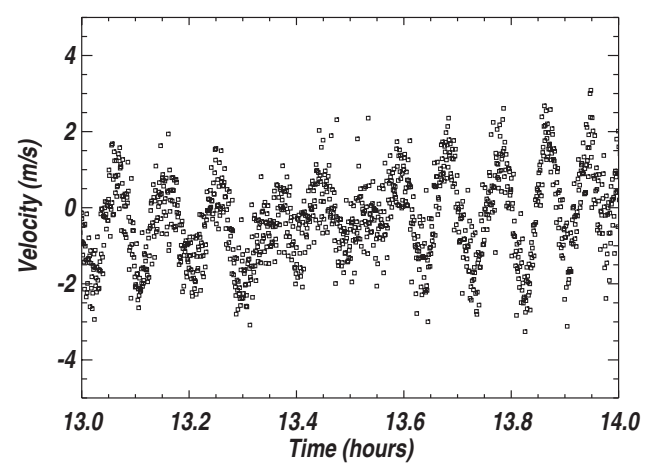

Figure 1. A one hour segment of solar data from June 11, 2012. The solar oscillations are clearly detected and the measured precision per data point $(0.5 \mathrm{~s}$ exposure $+2.3 \mathrm{~s}$ readout $)$ in this segment is close to $65 \mathrm{~cm} \mathrm{~s}^{-1}$. 
A more detailed account and comparison to dedicated solar instruments is presented by Pallé et al. (2013). This work showed that the measurements from SONG compare extremely well to simultaneous data from the well-characterised Mark-I instrument of the BiSON network (Brookeset al. 1978) and the GOLF (Gabriel et al. 1995) instrument on-board the SoHO $†$ spacecraft. This comparison showed that SONG has a very low noise at high frequencies - even lower than found by the Mark-I and GOLF instruments. While this points to excellent measurements, the lower noise is in part likely caused by differences in the details of how the three instruments measure the oscillations.

\section{2. $\beta$ Aquilae}

Following the initial telescope installation the sub-giant $\beta$ Aquilae was observed for four hours during July 11-12, 2012. The spectrograph employed a slit with a projected width of $1^{\prime \prime} .03$ - providing a spectral resolution of 90000 . The exposure time was $120 \mathrm{~s}$ for the first 100 spectra, and $180 \mathrm{~s}$ for the last 20 . Template observations of the star were also acquired during the same night. Corsaro et al. (2012) have previously provided a detailed study of $\beta$ Aquilae based on spectra from the SARG spectrograph at the Italian telescope TNG on La Palma (Gratton et al. 2001).

In Fig. 3 the radial velocity for the $4 \mathrm{~h}$ observing segment is shown. It is clear that $\beta$ Aquilae has a higher oscillation amplitude and lower frequency than the Sun. A direct comparison to the Corsaro et al. data shows that the amplitude and frequency is in excellent agreement with the SARG results (which were analysed using the same software).

\section{Towards other nodes}

While the prototype node is nearing completion at Observatorio del Teide, it is important to start network expansion - Arentoft et al. (2014) have shown the benefits of multiple sites for asteroseismic studies and how stars can be optimally selected in case of a 1, 2 or 3 -node network.

$\dagger$ Solar and Heliospheric Observatory

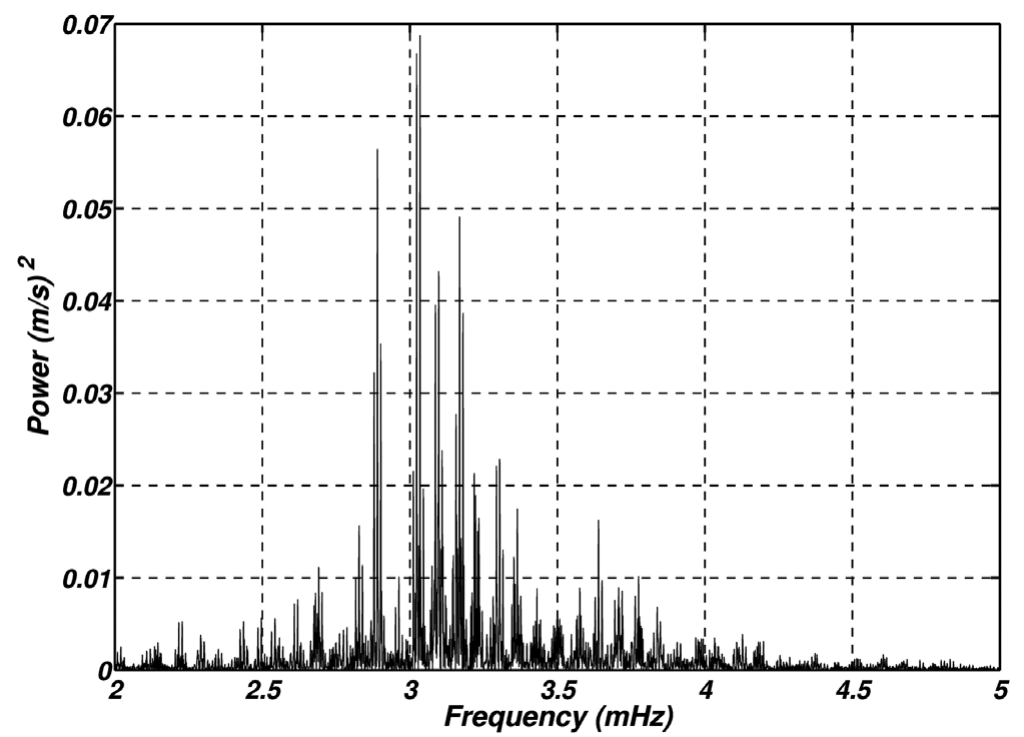

Figure 2. The resulting power spectrum for the 6-day solar observing series obtained June 11-16, 2012 from the Tenerife SONG node. 


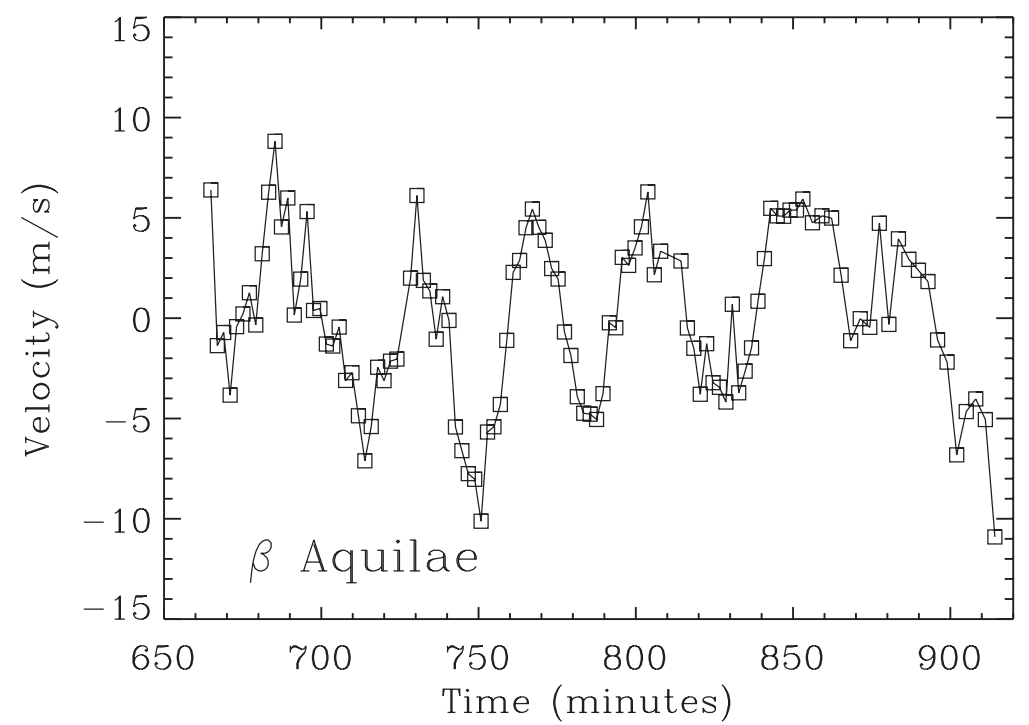

Figure 3. A $4^{\mathrm{h}}$ test sequence obtained for $\beta$ Aquilae in July 2012 with SONG.

Work on the installation of the second SONG node is nearly completed at the Delingha Observing Station in the Quinghai province in Chinaf with first light expected in late 2013. We anticipate that regular two-node network observations can begin around mid2014.

SONG is also actively pursuing funding possibilities for other nodes and hope to be successful in this endeavour within 1-2 years. To this end we plan to re-design the spectrograph pre-slit table to allow feeding light from the telescope via an optical fibre. This will allow the use of existing $1 \mathrm{~m}$-class telescopes to be used fully or partly as a SONG node. It is thus our hope that before the end of 2015 the northern half of SONG can be operational.

\section{Acknowledgements}

We gratefully acknowledge support from the Villum Foundation, the Carlsberg Foundation and Forskninsgrådet for Natur og Univers (FNU) towards the development of the SONG prototype. Funding for the Stellar Astrophysics Centre and the Centre for Star and Planet Formation is provided by The Danish National Research Foundation (Grant DNRF106 and grant DNRF97, respectively). The research is supported by the ASTERISK project (ASTERoseismic Investigations with SONG and Kepler) funded by the European Research Council (Grant agreement no.: 267864). Support from the Spanish Ministry of Economy and Competitiveness (MINECO, grant AYA2010-17803) is gratefully acknowledged.

\section{References}

Arentoft, T., Tingley, B., Christensen-Dalsgaard, J., Kjeldsen, H., White, T. R., \& Grundahl, F. 2014, MNRAS, 437, 1318

Baglin, A. \& Michel, E., CoRoT team 2012, ASP-CS, 462, 492

Brookes, J. R., Isaak, G. R., \& van der Raay, H. B. 1978, MNRAS, 185, 1

$\ddagger$ http://song.bao.ac.cn/ 
Butler, R. P., Marcy, G. W., Williams, E., McCarthy, C., \& Dosanjh, P., Vogt S. S. 1996, PASP, 108,500

Butler, R. P., Bedding, T. R., Kjeldsen, H., et al. 2004, ApJ, 600, L75

Buzasi, D. L. 2004, in: F. Favata, S. Aigrain, \& A. Wilson (eds.), Stellar Structure and Habitable Planet finding, ESA Special Publication, 538, 205

Chaplin, W. J. \& Miglio, A. 2013, ARAA, 51, 353

Corsaro, E., Grundahl, F., Leccia, S., Bonnano, A., Kjeldsen, H., \& Paternò, L. 2012, A\&ऽA, 537,8

Dziembowski, W. 1977, AcA, 27, 203

Gabriel, A. H., Grec, G., Charra, J., et al. 1995, Solar Phys., 162, 61

Gratton, R. G., Bonanno, G., Bruno, P., et al. 2001, Experimental Astronomy, 12, 107

Harpsøe, K. B. W., Andersen, M. I., \& Kjærgaard, P. 2012a, A $6 A$ A, 537, A50

Harpsøe, K. B. W., Jørgensen, U. G., Andersen, M. I., \& Grundahl, F. 2012b, A $\& A$, 542, A23

Harvey, J. W. 1988, in: J. Christensen-Dalsgaard, \& S. Frandsen (eds.), Advances in Helio- and Asteroseismology, IAU Symposium No. 123 (Dordrecht: D. Reidel Publishing Co.), p. 497

Kjeldsen, H., Bedding, T. R., Baldry, I. K., et al. 2003, AJ, 126, 1483

Matthews, J. M., Kuschnig, R., Guenther, D. B., et al. 2004, Nature, 430, 51

Pallé, P. L., Grundahl, F., Triviño Hage, A., et al. 2013, Journal of Physics, Conf. Series, 440, 012051

Triviño Hage, A., Uytterhoeven, K., Grundahl, F., \& Pallé, P. L., and the SONG team 2012, $A N 333,1107$ 